\title{
Covariance approach to the free photon field
}

\author{
Maciej Kuna \\ Wydział Fizyki Technicznej i Matematyki Stosowanej, \\ Politechnika Gdańska, \\ ul. Narutowicza 11/12, 80-952 Gdańsk, Poland \\ E-mail: maciek@mifgate.mif.pg.gda.pl \\ Jan Naudts \\ Departement Natuurkunde, Universiteit Antwerpen UIA, \\ Universiteitsplein 1, 2610 Antwerpen, Belgium \\ E-mail: Jan.Naudts@ua.ac.be
}

\begin{abstract}
We introduce photon theory following the same principles as for introduction of the quantum theory of a single particle, using a $C^{*}$-algebraic approach based on covariance systems. The basic symmetries are additivity of the fields and additivity of test functions. We write down in explicit form a state of this covariance system. It turns out to reproduce the traditional Fock representation of the free photon field, with a Lorentz invariant vacuum. Properties of smeared-out photons are discussed.
\end{abstract}

\section{Introduction}

\section{Motivation}

This paper is a first attempt to reformulate photon theory. It is motivated by dissatisfaction with expositions in present day textbooks. As Scharf[3] notes, the fact that there are various essentially different methods of quantizing the radiation field shows that there are some difficulties with the subject. Two problems must be recognized.

A first problem arises because of the use of the vector potential $A_{\mu}(q)$, which is not uniquely determined by the electromagnetic fields. The resulting gauge freedoms can be tackled in many ways. Most often used is the Gupta-Bleuler gauge, which is rather complicated to say the least. Disadvantage of the radiation gauge is the lack of manifest Lorentz covariance. Our treatment of the gauge problem has been influenced by the work of Carey et al[1], which uses the Lorenz gauge. We show that the photon states are invariant under the remaining gauge freedom. 
The next problem is that of positivity of the scalar product, in combination with Lorentz invariance. Many textbooks abandon the use of Hilbert spaces for this reason. Here, we give arguments to restrict the set of classical wave functions. A side effect is that the scalar product $\langle\psi \mid \phi\rangle$ of two classical wave functions $\phi$ and $\psi$ satisfies the positivity requirement. In addition, we prove explicitly in Appendix D that the vacuum state does satisfy the positivity condition. As a consequence, the quantum probabilistic interpretation is saved in our approach.

\section{Analogy}

The simplest example of a classical field is the vibrating string. Its canonical variables are a displacement field $\eta(q)$ and a conjugated momentum field $\pi(q)$. The correct description of the quantized string can be obtained by a limiting procedure starting from a chain of particles interconnected with strings. Alternatively, one can describe the quantized string as a covariance system[7] see Appendix A - consisting of an abelian algebra $\mathcal{A}$ generated by smeared-out displacement fields, a group $G$, which is the group of adding fields, and the obvious action of $G$ on $\mathcal{A}$. Such a description is very analogous to the description [9] of standard quantum mechanics as a covariance system, in which case $\mathcal{A}$ is an algebra of functions of position, and $G$ is the group of shifts in position. It is therefore natural to expect that also the quantized electromagnetic field can be described as a covariance system with additivity of fields as the basic symmetry group. The first problem that one encounters when trying such an approach is that the quantized smeared-out field operators $\hat{A}_{\mu}(\psi)$ do not form an abelian algebra. Indeed, in textbooks 2] one finds the commutation relations

$$
\left[\hat{A}_{\mu}(q), \hat{A}_{\nu}\left(q^{\prime}\right)\right]=-i g_{\mu, \nu} D_{0}\left(q-q^{\prime}\right)
$$

with the Pauli-Jordan function $D_{0}(q)$ defined by

$$
D_{0}(q)=\frac{1}{(2 \pi)^{3}} \int_{\mathbf{R}^{3}} \mathrm{~d} \mathbf{k} \exp \left(i \sum_{\alpha=1}^{3} \mathbf{k}_{\alpha} q_{\alpha}\right) \frac{1}{|\mathbf{k}|} \sin \left(q_{0}|\mathbf{k}|\right)
$$

(we have chosen the sign in (11) in such a way that later on creation and annihilation operators have their usual properties, i.e. the annihilation operator is complex linear in the field). Smearing out (11) with classical wave functions $\psi$ and $\phi$ gives (see Appendix B)

$$
[\hat{A}(\psi), \hat{A}(\phi)]=2 i \operatorname{Im} \int_{\mathbf{R}^{3}} \mathrm{~d} \mathbf{k} \frac{1}{2|\mathbf{k}|} \overline{\psi^{\mu}(\mathbf{k})} \phi_{\mu}(\mathbf{k}) .
$$

There are two possible interpretations of these non-trivial commutation relations. It is a tradition in the physics literature to interpret photons as excitations of a harmonic oscillator. In this traditional approach[2, the canonical variables to be quantized are the vector fields $A_{\mu}(q)$ and their derivatives 
$\delta_{\nu} A_{\mu}(q)$. These correspond with the displacement field $\eta(q)$ and conjugated momentum field $\pi(q)$ of the harmonic string. By integration, one then obtains (3). An alternative interpretation is suggested by recent research on noncommutative spacetime. In the latter context, spacetime positions $\hat{Q}_{\mu}$ satisfy nontrivial commutation relations

$$
\left[\hat{Q}_{\mu}, \hat{Q}_{\nu}\right]=i \epsilon_{\mu \nu}
$$

(see e.g. Doplicher et al[5, 6] and Naudts and Kuna [8]). The $\hat{Q}_{\mu}$ are the generators of shifts in momentum space. The commutation relations (4) can [8] be seen as a originating from a projective representation of the group of shifts. By analogy, we can see the operators $\hat{A}(\psi)$ as generators of the group of addition of classical wave functions. In fact, it is clear[9] that the Weyl algebra of the free photon field, as used e.g. in Carey et al[1], can be replaced by a covariance system $(\mathbf{C}, H, \mathbf{I})$ consisting of the algebra $\mathbf{C}$ of complex numbers, the complex vector space of classical wave functions $H$, and the trivial action $\mathbf{I}$ of the latter on $\mathbf{C}$.

\section{Duality}

Because the addition of fields is the basic symmetry, rather than addition of classical wave functions, it is obvious to consider also the generators $\hat{F}(a)$ of the group of adding fields

$$
a^{\prime}(\mathbf{k}) \rightarrow a^{\prime}(\mathbf{k})+a(\mathbf{k}) .
$$

Here, the fields are represented by Fourier coefficients $a_{\mu}(\mathbf{k})$ of the vector potential $A_{\mu}(q)$ (see next section). In this context a duality between classical wave functions and Fourier transformed vector potentials is of importance. It implies a duality between the operators $\hat{A}(\psi)$ and $\hat{F}(a)$, similar to the duality between position and momentum operators in standard quantum mechanics.

Technically speaking, there is no need to include this duality in the formalism. Indeed, we will find that, in the Fock representation, for each $a$ there exists a $\psi$ such that $\hat{F}(a)=\hat{A}(\psi)$. However, this coincidence is a special property of the photon field and might be absent in more general field theories. For that reason we prefer to clarify the dual rôle of the operators $\hat{A}(\psi)$ and $\hat{F}(a)$.

\section{Notations}

We use Greek letters $\mu, \nu, \sigma, \cdots$ for indices that run from 0 to 3 , in combination with Einstein's summing convention, i.e., if such an index appears twice then a summation from 0 to 3 is understood. These Greek indices are lowered and raised in the standard way, i.e., by definition is $x^{\mu}=g^{\mu \nu} x_{\nu}$, with the metric tensor $g$ equal to the diagonal matrix with eigenvalues $+1,-1,-1,-1$. The Greek index $\alpha$ will be used to label spatial components. Hence it runs from 1 to 3 and no summation convention is used for it. Vectors in $\mathbf{R}^{3}$ are written in boldface. Quite often, a four-vector $q$ will be written as $\left(q_{0}, \mathbf{q}\right)$. The scalar 
product in $\mathbf{R}^{3}$ is written as $\mathbf{k} \cdot \mathbf{q}=\sum_{\alpha=1}^{3} \mathbf{k}_{\alpha} \mathbf{q}_{\alpha}$. We use the abbreviation $\partial^{\mu} \equiv \frac{\partial}{\partial q_{\mu}}$.

The (pseudo)-scalar product of two elements $\psi$ and $\phi$ of a (pseudo)-Hilbert space is denoted $\langle\phi \mid \psi\rangle$, linear in $\psi$ and anti-linear in $\phi$. Throughout the paper, operators carry a hat. The conjugate of $\hat{A}$ is denoted $\hat{A}^{*}$.

\section{Structure of the paper}

The next section deals with classical electromagnetism. The vector potential $A_{\mu}(q)$ is represented by Fourier coefficients $a_{\mu}(\mathbf{k})$. The classical wave functions $\psi_{\mu}(\mathbf{k})$ are introduced and the duality between classical wave functions $\psi_{\mu}(\mathbf{k})$ and Fourier coefficients $a_{\mu}(\mathbf{k})$ is established. Section 3 describes the free photon field as a covariance system. Correlation functions determining the vacuum state are given explicitly. The field operators $\hat{A}(\psi)$ and $\hat{F}(a)$ live in the corresponding G.N.S.-representation. Section 1 discusses the standard Fock representation of the free photon field. Section 5 starts from Poincaré invariance to derive properties of the free photon. In the final section conclusions are drawn.

\section{Classical electromagnetism}

The whole section deals with the classical radiation field. The vector potential $A_{\mu}(q)$ is replaced by Fourier coefficients $a_{\mu}(\mathbf{k})$. The test functions $f_{\mu}(q)$, used to smear out the vector potential $A_{\mu}(q)$, are replaced by the classical wave functions $\psi_{\mu}(\mathbf{k})$. Finally, a duality between $a_{\mu}(\mathbf{k})$ and $\psi_{\mu}(\mathbf{k})$ is established.

\section{Smeared-out fields}

The classical electromagnetic field is described by the vector potential $A(q)$. It has four components $A_{\mu}(q), \mu=0,1,2,3$, each of which is a function of position $q$ in $\mathbf{R}^{4}$. We assume that the Lorenz gauge

$$
\partial^{\mu} A_{\mu}(q)=0
$$

is satisfied. Then the Maxwell equations for the free electromagnetic field can be written as a set of four equations

$$
\partial^{\nu} \partial_{\nu} A_{\mu}(q)=0 .
$$

It is necessary to smear out $A$ using test functions. Given real-valued test functions $f_{\mu}(q)$, let

$$
f(A)=\int_{\mathbf{R}^{4}} \mathrm{~d} q f^{\mu}(q) A_{\mu}(q)
$$

The functions $f(A)$ will become observables of the free photon field. 
The electric field $\mathbf{E}$ and the magnetic field $\mathbf{B}$ are related to the vector potential $A$ by

$$
\begin{aligned}
& \mathbf{E}_{\alpha}(q)=-\partial^{\alpha} A_{0}(q)-\partial^{0} A_{\alpha}, \\
& \mathbf{B}_{\alpha}(q)=\sum_{\beta, \gamma=1}^{3} \varepsilon_{\alpha \beta \gamma} \partial^{\beta} A_{\gamma}(q)
\end{aligned}
$$

( $\varepsilon_{\alpha \beta \gamma}$ is the fundamental antisymmetric tensor).

Note that the smeared-out electromagnetic fields can be obtained from the smeared-out vector potential. Indeed one has

$$
\begin{aligned}
\int_{\mathbf{R}^{4}} \mathrm{~d} q \sum_{\alpha=1}^{3} f_{\alpha}(q) \mathbf{E}_{\alpha}(q)= & -\int_{\mathbf{R}^{4}} \mathrm{~d} q \sum_{\alpha=1}^{3} f_{\alpha}(q) \partial^{\alpha} A_{0}(q) \\
& -\int_{\mathbf{R}^{4}} \mathrm{~d} q \sum_{\alpha=1}^{3} f_{\alpha}(q) \partial^{0} A_{\alpha}(q) \\
= & \int_{\mathbf{R}^{4}} \mathrm{~d} q A_{0}(q) \sum_{\alpha=1}^{3} \partial^{\alpha} f_{\alpha}(q) \\
& +\int_{\mathbf{R}^{4}} \mathrm{~d} q \sum_{\alpha=1}^{3} A_{\alpha}(q) \partial^{0} f_{\alpha}(q) \\
= & g(A)
\end{aligned}
$$

with

$$
g_{0}(q)=\sum_{\alpha=1}^{3} \partial^{\alpha} f_{\alpha}(q) \text { and } g_{\alpha}(q)=-\partial^{0} f_{\alpha}(q),
$$

and, similarly,

$$
\begin{aligned}
\int_{\mathbf{R}^{4}} \mathrm{~d} q \sum_{\alpha=1}^{3} f_{\alpha}(q) \mathbf{B}_{\alpha}(q) & =\int_{\mathbf{R}^{4}} \mathrm{~d} q \sum_{\alpha=1}^{3} f_{\alpha}(q) \sum_{\beta, \gamma=1}^{3} \varepsilon_{\alpha \beta \gamma} \partial^{\beta} A_{\gamma}(q) \\
& =-\int_{\mathbf{R}^{4}} \mathrm{~d} q \sum_{\alpha, \beta, \gamma=1}^{3} \varepsilon_{\alpha \beta \gamma}\left(\partial^{\beta} f_{\alpha}(q)\right) A_{\gamma}(q) \\
& =h(A)
\end{aligned}
$$

with

$$
h_{0}(q)=0 \quad \text { and } \quad h_{\gamma}(q)=\sum_{\alpha, \beta=1}^{3} \varepsilon_{\alpha \beta \gamma} \partial^{\beta} f_{\alpha}(q)
$$

\section{Fourier coefficients}

Equation (7) can be solved by Fourier transformation. Let

$$
\tilde{A}_{\mu}(k)=(2 \pi)^{-2} \int_{\mathbf{R}^{4}} \mathrm{~d} q \exp \left(i k^{\nu} q_{\nu}\right) A_{\mu}(q) .
$$


Then (7) becomes $k^{\nu} k_{\nu} \tilde{A}_{\mu}(k)=0$. Hence $\tilde{A}_{\mu}(k)$ differs from zero only if $k^{\nu} k_{\nu}=$ 0 . Therefore $A_{\mu}$ is of the form

$$
\begin{aligned}
A_{\mu}(q)= & (2 \pi)^{-2} \int_{\mathbf{R}^{4}} \mathrm{~d} k \exp \left(-i k^{\nu} q_{\nu}\right) \tilde{A}_{\mu}(k) \delta\left(k^{\sigma} k_{\sigma}\right) \\
= & (2 \pi)^{-2} \int_{\mathbf{R}^{4}} \mathrm{~d} k \exp \left(-i k^{\nu} q_{\nu}\right) \tilde{A}_{\mu}(k) \frac{1}{2|\mathbf{k}|}\left(\delta\left(k^{0}-|\mathbf{k}|\right)+\delta\left(k^{0}+|\mathbf{k}|\right)\right) \\
= & (2 \pi)^{-2} \int_{\mathbf{R}^{3}}^{\mathrm{d}} \mathrm{d} \frac{1}{2|\mathbf{k}|} \exp (i \mathbf{k} \cdot \mathbf{q}) \\
& \times\left(e^{-i|\mathbf{k}| q_{0}} \tilde{A}_{\mu}(|\mathbf{k}|, \mathbf{k})+e^{i|\mathbf{k}| q_{0}} \tilde{A}_{\mu}(-|\mathbf{k}|, \mathbf{k})\right)
\end{aligned}
$$

Here we use the notation $|\mathbf{k}|=\sqrt{\sum_{\alpha=1}^{3} k_{\alpha}^{2}}$. We obtain

$$
A_{\mu}(q)=(2 \pi)^{-3 / 2} \int_{\mathbf{R}^{3}} \mathrm{~d} \mathbf{k} \frac{1}{2|\mathbf{k}|} e^{i \mathbf{q} \cdot \mathbf{k}}\left[e^{-i q_{0}|\mathbf{k}|} a_{\mu}(\mathbf{k})+e^{i q_{0}|\mathbf{k}|} \overline{a_{\mu}(-\mathbf{k})}\right]
$$

with $a_{\mu}(\mathbf{k})=(2 \pi)^{-1 / 2} \tilde{A}_{\mu}(|\mathbf{k}|, \mathbf{k})$. Note that automatically any vector potential of the form (16) satisfies the wave equations (7). Expression (8), in combination with (16), becomes

$$
f(A)=\sqrt{2 \pi} \int_{\mathbf{R}^{3}} \mathrm{~d} \mathbf{k} \frac{1}{2|\mathbf{k}|}\left[a^{\mu}(\mathbf{k}) \overline{\tilde{f}_{\mu}(|\mathbf{k}|, \mathbf{k})}+\overline{a^{\mu}(\mathbf{k})} \tilde{f}_{\mu}(|\mathbf{k}|, \mathbf{k})\right]
$$

with

$$
\tilde{f}_{\mu}(k)=(2 \pi)^{-2} \int_{\mathbf{R}^{4}} \mathrm{~d} q \exp \left(i k^{\nu} q_{\nu}\right) f_{\mu}(q) .
$$

Note that only the values of $\tilde{f}_{\mu}$ on the light cone $\left\{k \in \mathbf{R}^{4}: k^{\mu} k_{\mu}=0\right\}$ are of importance.

From the Lorenz condition (6) follows

$$
|\mathbf{k}| a_{0}(\mathbf{k})=\sum_{\alpha=1}^{3} \mathbf{k}_{\alpha} a_{\alpha}(\mathbf{k}) .
$$

This expression can be used to calculate $a_{0}(\mathbf{k})$ in function of $a_{\alpha}(\mathbf{k})$.

\section{Classical wave functions}

The first gauge problem that arises is that two different sets of test functions $f_{\mu}(q)$ and $g_{\mu}(q)$ may define functions $f(A)$ and $g(A)$ which coincide on all vector potentials $A$ that satisfy (6) and (7). To avoid this non-uniqueness we make use of the so-called classical wave functions of the photon. Given test functions $f_{\mu}$ the classical wave functions $\psi_{\mu}$ are defined by

$$
\psi_{\mu}(\mathbf{k})=\sqrt{2 \pi} \tilde{f}_{\mu}(|\mathbf{k}|, \mathbf{k})
$$




$$
=(2 \pi)^{-3 / 2} \int_{\mathbf{R}^{4}} \mathrm{~d} q \exp \left(i q_{0}|\mathbf{k}|-i \mathbf{q} \cdot \mathbf{k}\right) f_{\mu}(q) .
$$

Note that these are complex functions over $\mathbf{R}^{3}$. Two sets of test functions $f_{\mu}$ and $g_{\mu}$ can give rise to the same classical wave functions $\psi_{\mu}$. In fact, this will be the case if and only if $f(A)=g(A)$ for all $A$ satisfying (6) and (7). Indeed, (17) can be written as

$$
f(A)=\int_{\mathbf{R}^{3}} \mathrm{~d} \mathbf{k} \frac{1}{2|\mathbf{k}|}\left[a^{\mu}(\mathbf{k}) \overline{\psi_{\mu}(\mathbf{k})}+\overline{a^{\mu}(\mathbf{k})} \psi_{\mu}(\mathbf{k})\right]=-2 \operatorname{Re}\langle a \mid \psi\rangle
$$

where the bilinear form $\langle\cdot \mid \cdot\rangle$ is given by

$$
\langle a \mid \psi\rangle=-\int_{\mathbf{R}^{3}} \mathrm{~d} \mathbf{k} \frac{1}{2|\mathbf{k}|} \overline{a^{\mu}(\mathbf{k})} \psi_{\mu}(\mathbf{k}) .
$$

This shows that $f(A)$ depends only on the classical wave functions $\psi$ and on the Fourier coefficients $a$.

\section{Duality}

The Lorenz gauge (6) does not suffice to fix uniquely the vector potential $A$ corresponding with a given electromagnetic field. The gauge transformations $A_{\mu} \rightarrow A_{\mu}^{\prime}$ with

$$
A_{\mu}^{\prime}=A_{\mu}+\partial_{\mu} \chi
$$

with $\chi(q)$ an arbitrary solution of $\partial^{\mu} \partial_{\mu} \chi=0$ leave the electric and magnetic fields invariant. We use this gauge freedom to derive a condition on the classical wave functions. If two fields $A$ and $A^{\prime}$ differ only by a gauge transformation as given by (23), then no classical wave function $\psi$ should be able to distinguish $A$ from $A^{\prime}$, i.e. $\operatorname{Re}\langle a \mid \psi\rangle=\operatorname{Re}\left\langle a^{\prime} \mid \psi\right\rangle$ should hold. The result of this condition, deduced below, is

$$
|\mathbf{k}| \psi_{0}(\mathbf{k})=\sum_{\alpha=1}^{3} \mathbf{k}_{\alpha} \psi_{\alpha}(\mathbf{k}) .
$$

This condition implies a duality between Fourier coefficients $a(\mathbf{k})$ determining the vector potential $A$ and classical wave functions $\psi(\mathbf{k})$ determining test functions $f$. Indeed, both are sets of four complex functions satisfying similar conditions (19) respectively (24).

Because $\chi$ is a solution of $\partial^{\mu} \partial_{\mu} \chi=0$ it can be written as (see (16))

$$
\chi(q)=\int_{\mathbf{R}^{3}} \mathrm{~d} \mathbf{k} \frac{1}{2|\mathbf{k}|} e^{i \mathbf{q} \cdot \mathbf{k}}\left[e^{-i q_{0}|\mathbf{k}|} c(\mathbf{k})+e^{i q_{0}|\mathbf{k}|} \overline{c(-\mathbf{k})}\right],
$$

with $c(\mathbf{k})$ an arbitrary complex function of $\mathbf{k} \in \mathbf{R}^{3}$. From (23) and (16) follows then that

$$
a_{\alpha}^{\prime}(\mathbf{k})=a_{\alpha}(\mathbf{k})+i c(\mathbf{k}) \mathbf{k}_{\alpha}, \quad \alpha=1,2,3
$$


Using (22) and (19) one obtains

$$
\begin{aligned}
\langle a \mid \psi\rangle & =-\int_{\mathbf{R}^{3}} \mathrm{~d} \mathbf{k} \frac{1}{2|\mathbf{k}|}\left(\overline{a_{0}(\mathbf{k})} \psi_{0}(\mathbf{k})-\sum_{\alpha=1}^{3} \overline{a_{\alpha}(\mathbf{k})} \psi_{\alpha}(\mathbf{k})\right) \\
& =-\int_{\mathbf{R}^{3}} \mathrm{~d} \mathbf{k} \frac{1}{2|\mathbf{k}|^{2}} \sum_{\alpha=1}^{3} \overline{a_{\alpha}(\mathbf{k})}\left(\psi_{0}(\mathbf{k}) \mathbf{k}_{\alpha}-|\mathbf{k}| \psi_{\alpha}(\mathbf{k})\right)
\end{aligned}
$$

and a similar expression for $\left\langle a^{\prime} \mid \psi\right\rangle$. Hence the condition $\operatorname{Re}\left\langle a^{\prime} \mid \psi\right\rangle=\operatorname{Re}\langle a \mid \psi\rangle$ yields

$$
0=\operatorname{Re} \int_{\mathbf{R}^{3}} \mathrm{~d} \mathbf{k} \frac{1}{2|\mathbf{k}|^{2}} \sum_{\alpha=1}^{3} i \mathbf{k}_{\alpha} \overline{c(\mathbf{k})}\left(\psi_{0}(\mathbf{k}) \mathbf{k}_{\alpha}-|\mathbf{k}| \psi_{\alpha}(\mathbf{k})\right) .
$$

Because the latter should hold for all choices of $c(\mathbf{k})$ one concludes that (24) holds.

\section{Radiation gauge}

Note that the scalar product $\langle a \mid \psi\rangle$ is degenerate. Indeed, condition (24), to be satisfied by classical wave functions, was derived precisely by requiring that, if a gauge transformation maps $a$ onto $b$ then $\langle a \mid \psi\rangle=\langle b \mid \psi\rangle$ holds for all $\psi$. By duality, we say that $\psi$ and $\phi$ are equivalent if $\langle a \mid \psi\rangle=\langle a \mid \phi\rangle$ holds for all $a$. As noted by Carey et al [1], in each set of equivalent $\psi$ one can select a unique representative $\psi$ satisfying

$$
\psi_{0}(\mathbf{k})=0 \quad \text { and } \quad \sum_{\alpha=1}^{3} \mathbf{k}_{\alpha} \psi_{\alpha}(\mathbf{k})=0 .
$$

See Appendix C. These conditions are called the radiation gauge. However, by selecting such a representative one breaks the property of manifest Lorentz invariance. Therefore, we will use this gauge only to discuss the physical content of certain formulas.

\section{Quantum description}

This section gives a description of the electromagnetic field as a quantum system. We start from explicit correlation functions and use the generalized GNStheorem to construct a representation in Hilbert space.

\section{Covariance approach}

In the previous section the smeared-out vector potential $f(A)$ could be written as $-2 \operatorname{Re}\langle a \mid \psi\rangle$ (see (21)), where $a(\mathbf{k})$ are Fourier coefficients representing the vector potential $A_{\mu}(q)$ and $\psi(\mathbf{k})$ is a classical wave function representing the test 
functions $f_{\mu}(q)$. In the quantum theory both $a(\mathbf{k})$ and $\psi(\mathbf{k})$ become operators in Hilbert space. They will be denoted $\hat{F}(a)$ and $\hat{A}(\psi)$, respectively.

We want to derive the quantum description of the electromagnetic field in a way similar to the quantum description of a single particle. The quantity corresponding with a function $f(q)$ of the position $q$ of the particle is the function $f(A)$ considered as a function of the vector potential $A_{\mu}$. Hence, in the obvious quantum description quantum mechanical wave functions would be complex square integrable functions of $A_{\mu}$ (replacing $q$-dependent functions) and the $f(A)$ is mapped onto an operator $f(\hat{A})$ (replacing $f(\hat{q})$, with $\hat{q} \psi(q)=q \psi(q)$ ). A more common notation, replacing $f(\hat{A})$, is $\hat{A}(\psi)$, with $\psi$ the classical wave function corresponding with $f$. As discussed in the introduction, the problem with this approach is that the operators $\hat{A}(\psi)$ are expected not to be mutually commuting, so that they cannot be simple multiplication operators, as in the case of quantum mechanics of a single particle. The solution adopted here is to see the operators $\hat{A}(\psi)$ as generators of the group of adding test functions. An additional advantage of this point of view is that it is then natural to consider also the group of adding fields. The generators of the latter group are the operators $\hat{F}(a)$. Another advantage is that the resulting formalism is very close to the $C^{*}$-algebraic approach using Weyl algebras㑛,

\section{Correlation functions}

The classical wave functions $\psi$ form a linear space, denoted $H$. The Fourier coefficients $a$ belong to the dual space $H^{*}$. In what follows we will consider $H^{*}$ as a real linear space, and not a complex one, because only multiplication of $a_{\mu}$ with a real constant corresponds with multiplication of the vector potential $A_{\mu}$ with the same constant. As a consequence, also $H$ will be considered as a real linear space.

Consider $H^{*} \times H$ as an additive group. A state of the covariance system $\left(\mathbf{C}, H^{*} \times H, \mathbf{I}\right)$ is determined by correlation functions $\mathcal{F}(a, \psi ; b, \phi)$. We make the following choice:

$$
\begin{aligned}
\mathcal{F}(a, \psi ; b, \phi) & =\exp \left(-\frac{i}{2 \eta} \operatorname{Im}\langle b+i \eta \phi \mid a+i \eta \psi\rangle\right) \\
& \times \exp \left(-\frac{1}{4 \eta}\langle b-a+i \eta(\phi-\psi) \mid b-a+i \eta(\phi-\psi)\rangle\right)
\end{aligned}
$$

with $\eta$ a positive number. The proof that these correlation functions have the necessary properties to define a state of $\left(\mathbf{C}, H^{*} \times H, \mathbf{I}\right)$ is given in Appendix D. The generalized GNS-theorem[7] implies that there exists a projective representation $\hat{W}(a, \psi)$ of $H^{*} \times H$ in a Hilbert space $\mathcal{H}$, and a normalized wave function $\Omega$ in $\mathcal{H}$, for which

$$
\mathcal{F}(a, \psi ; b, \phi)=\left\langle\hat{W}(b, \phi)^{*} \Omega \mid \hat{W}(a, \psi)^{*} \Omega\right\rangle
$$

holds. 


\section{The cocycle}

From the ansatz

$$
\hat{W}(a, \psi) \hat{W}(b, \phi)=\exp \left(\frac{i}{2} s(a, \psi ; b, \phi)\right) \hat{W}(a+b, \psi+\phi)
$$

follows, using that $\hat{W}(a, \psi)^{*}=\hat{W}(-a,-\psi)$,

$$
\begin{aligned}
\mathcal{F}(a, \psi ; b, \phi) & =\langle\Omega \mid \hat{W}(b, \phi) \hat{W}(-a,-\psi) \Omega\rangle \\
& =\exp \left(-\frac{i}{2} s(b, \phi ; a, \psi)\right)\langle\Omega \mid \hat{W}(b-a, \phi-\psi) \Omega\rangle \\
& =\exp \left(-\frac{i}{2} s(b, \phi ; a, \psi)\right) \mathcal{F}(a-b, \psi-\phi ; 0,0)
\end{aligned}
$$

Using the definition of $\mathcal{F}$ one obtains

$$
s(a, \psi ; b, \phi)=\eta^{-1} \operatorname{Im}\langle a+i \eta \psi \mid b+i \eta \phi\rangle .
$$

This function $s(a, \psi ; b, \phi)$ is a symplectic form, as it should be. It is antisymmetric under exchange of $(a, \psi)$ and $(b, \phi)$. It is real linear in its arguments. Note that it is degenerate because $\langle\cdot \mid \cdot\rangle$ is degenerate. The function $\exp ((i / 2) s(a, \psi ; b, \phi))$, appearing in 32$)$, is a cocycle of the additive group $H^{*} \times H$.

\section{Field operators}

The operators $\hat{A}(\psi)$ and $\hat{F}(a)$ are introduced as self-adjoint operators satisfying

$$
\hat{W}(0, \lambda \psi)=\exp (i \lambda \hat{A}(\psi)) \quad \text { and } \quad \hat{W}(\lambda a, 0)=\exp (i \lambda \hat{F}(a))
$$

for all real $\lambda$. The commutation relations for these operators can be obtained from

$$
\hat{W}(a, \psi) \hat{W}(b, \phi)=\exp (i s(a, \psi ; b, \phi)) \hat{W}(b, \phi) \hat{W}(a, \psi)
$$

by inserting real numbers, as in (35), and taking derivatives. One obtains

$$
\begin{aligned}
{[\hat{F}(a), \hat{F}(b)] } & =-i \eta^{-1} \operatorname{Im}\langle a \mid b\rangle \\
{[\hat{F}(a), \hat{A}(\phi)] } & =-i \operatorname{Re}\langle a \mid \phi\rangle \\
{[\hat{A}(\psi), \hat{A}(\phi)] } & =-i \eta \operatorname{Im}\langle\psi \mid \phi\rangle .
\end{aligned}
$$

Comparison with the traditional result (3) gives $\eta=2$.

The unitary operator $\hat{W}(a)$ implements adding (or subtracting) a field. Indeed, one calculates

$$
\hat{W}(a, 0) \hat{A}(\psi) \hat{W}(-a, 0)=-\left.i \frac{\mathrm{d}}{\mathrm{d} \lambda}\right|_{\lambda=0} \hat{W}(a, 0) \hat{W}(0, \lambda \psi) \hat{W}(-a, 0)
$$




$$
\begin{aligned}
& =-\left.i \frac{\mathrm{d}}{\mathrm{d} \lambda}\right|_{\lambda=0} \exp (i \lambda s(a, 0 ; 0, \psi)) \hat{W}(0, \lambda \psi) \\
& =\hat{A}(\psi)+\operatorname{Re}\langle a \mid \psi\rangle \\
& =\hat{A}(\psi)-f(A)
\end{aligned}
$$

with $f_{\mu}(q)$ the test functions corresponding with $\psi$ and $A_{\mu}(q)$ the vector potential corresponding with $a$. Formally, this can be rewritten as

$$
\hat{W}(a, 0) \hat{A}_{\mu}(q) \hat{W}(-a, 0)=\hat{A}_{\mu}(q)-A_{\mu}(q) .
$$

Similarly, the unitary operator $\hat{W}(0, \psi)$ implements adding wave functions. Indeed, one finds

$$
\begin{aligned}
\hat{W}(0, \psi) \hat{F}(a) \hat{W}(0,-\psi) & =\hat{F}(a)-\operatorname{Re}\langle\psi \mid a\rangle \\
& =\hat{F}(a)+f(A) .
\end{aligned}
$$

\section{Real additivity and identification}

Let us calculate

$$
\begin{aligned}
& \left\langle\hat{W}(b, \phi)^{*} \Omega \mid \hat{A}(\chi) \hat{W}(a, \psi)^{*} \Omega\right\rangle \\
& =\left.i \frac{\partial}{\partial \lambda}\right|_{\lambda=0}\left\langle\hat{W}(b, \phi)^{*} \Omega \mid \hat{W}(0, \lambda \chi)^{*} \hat{W}(a, \psi)^{*} \Omega\right\rangle \\
& =\left.i \frac{\partial}{\partial \lambda}\right|_{\lambda=0} ^{\lambda=0} \exp (-(i \lambda / 2) \operatorname{Re}\langle\chi \mid a+i \eta \psi\rangle) \mathcal{F}(a, \psi+\lambda \chi ; b, \phi) \\
& =\frac{1}{2}[\langle a+i \eta \psi \mid \chi\rangle+\langle\chi \mid b+i \eta \phi\rangle]\left\langle\hat{W}(b, \phi)^{*} \Omega \mid \hat{W}(a, \psi)^{*} \Omega\right\rangle
\end{aligned}
$$

and, similarly,

$$
\begin{aligned}
& \left\langle\hat{W}(b, \phi)^{*} \Omega \mid \hat{F}(d) \hat{W}(a, \psi)^{*} \Omega\right\rangle \\
= & \frac{i}{2 \eta}[\langle d \mid b+i \eta \phi\rangle-\langle a+i \eta \psi \mid d\rangle]\left\langle\hat{W}(b, \phi)^{*} \Omega \mid \hat{W}(a, \psi)^{*} \Omega\right\rangle .
\end{aligned}
$$

These expressions show that the operators $\hat{A}(\chi)$ and $\hat{F}(d)$ are real linear functions. Moreover, comparison of the two expressions yields $\eta \hat{F}(i \chi)=\hat{A}(\chi)$ for all $\chi$. One concludes that in the Hilbert space representation determined by the correlation functions (30) the generators of adding fields, respectively of adding wave functions, coincide.

\section{Fock representation}

In this section the Hilbert space representation determined by the correlation functions (30) is identified with the Fock space in which photon states are created by repeated application of creation operators onto the vacuum state. 


\section{Creation and annihilation operators}

From (41) follows that

$$
\left\langle\hat{W}(b, \phi)^{*} \Omega \mid \hat{A}(\psi) \Omega\right\rangle=\frac{1}{2}\left\langle\hat{W}(b, \phi)^{*} \Omega \mid \Omega\right\rangle\langle\psi \mid b+i \eta \phi\rangle .
$$

Hence one has

$$
\left\langle\hat{W}(b, \phi)^{*} \Omega \mid(\hat{A}(\psi)-i \hat{A}(i \psi)) \Omega\right\rangle=0 .
$$

Since $b$ and $\phi$ are arbitrary this implies that

$$
(\hat{A}(\psi)-i \hat{A}(i \psi)) \Omega=0 .
$$

It is therefore obvious to define annihilation operators $\hat{A}_{-}(\psi)$ by

$$
\hat{A}_{-}(\psi)=\frac{1}{2} \hat{A}(\psi)-\frac{i}{2} \hat{A}(i \psi),
$$

which means that also

$$
\hat{A}_{-}(\psi)=\frac{1}{2} \hat{A}(\psi)+\frac{i \eta}{2} \hat{F}(\psi) .
$$

The latter expression resembles the definition $Q+i P$ of the annihilation operator by means of a pair of position and momentum operators, in the context of the harmonic oscillator. Note that $\hat{A}_{-}(\psi)$ is a complex linear function of $\psi$. As shown above, the annihilation operators satisfy

$$
\hat{A}_{-}(\psi) \Omega=0 .
$$

One verifies that these operators are commuting

$$
\left[\hat{A}_{-}(\psi), \hat{A}_{-}(\phi)\right]=0
$$

The conjugate operator $\hat{A}_{+}(\psi)=\hat{A}_{-}(\psi)^{*}$ is the creation operator. From the definition follows immediately that

$$
\hat{A}(\psi)=\hat{A}_{+}(\psi)+\hat{A}_{-}(\psi) .
$$

The commutation relations between creation and annihilation operators are found to be

$$
\begin{aligned}
{\left[\hat{A}_{+}(\psi), \hat{A}_{-}(\phi)\right] } & =\frac{1}{4}[\hat{A}(\psi)+i \hat{A}(i \psi), \hat{A}(\phi)-i \hat{A}(i \phi)] \\
& =-\frac{\eta}{2}\langle\psi \mid \phi\rangle .
\end{aligned}
$$

With $\eta=2$ this relation gives to the operator $\hat{A}_{+}(\psi) \hat{A}_{-}(\phi)$ the usual interpretation of number operator. 


\section{One-photon states}

A one-photon state is determined by an element of the Hilbert space of the form $\hat{A}_{+}(\xi) \Omega$, where $\xi$ is a classical wave function, not equivalent to zero. It is straightforward to verify that two equivalent classical wave functions $\psi$ and $\phi$ determine the same one-photon state. Indeed, by definition they satisfy $\langle a \mid \psi\rangle=$ $\langle a \mid \phi\rangle$ for all $a$. From (43) then follows that $\hat{A}(\psi) \Omega=\hat{A}(\phi) \Omega$ so that $\hat{A}_{+}(\psi) \Omega=$ $\hat{A}_{+}(\phi) \Omega$. Hence $\phi$ and $\psi$ determine the same wave function in the Hilbert space, and hence, the same physical state.

A short calculation gives

$$
\begin{aligned}
\left\|\hat{A}_{+}(\psi) \Omega\right\|^{2} & =\left\langle\Omega \mid \hat{A}_{-}(\psi) \hat{A}_{+}(\psi) \Omega\right\rangle \\
& =-\left\langle\Omega \mid\left[\hat{A}_{+}(\psi), \hat{A}_{-}(\psi)\right] \Omega\right\rangle \\
& =\frac{\eta}{2}\langle\psi \mid \psi\rangle \\
& =\frac{\eta}{2} \int_{\mathbf{R}^{3}} \mathrm{~d} \mathbf{k} \frac{1}{2|\mathbf{k}|^{3}} \sum_{\alpha, \beta=1}^{3} \overline{\psi_{\alpha}(\mathbf{k})}\left(|\mathbf{k}|^{2} \delta_{\alpha \beta}-\mathbf{k}_{\alpha} \mathbf{k}_{\beta}\right) \psi_{\beta}(\mathbf{k}) \\
& \geq 0 .
\end{aligned}
$$

The last steps of this calculation use results of Appendix D. In particular, $\left\|\hat{A}_{+}(\psi) \Omega\right\|=0$ holds if $\psi_{\mu}(\mathbf{k})$ is of the form

$$
\psi_{\alpha}(\mathbf{k})=\frac{\mathbf{k}_{\alpha}}{|\mathbf{k}|} \psi_{0}(\mathbf{k}), \quad \alpha=1,2,3 .
$$

The usual interpretation of this result is that there do not exist photon states for which the electric and magnetic fields are not perpendicular to the wave vector $\mathbf{k}$.

\section{Poincaré invariance}

In this section we study the action of the proper Poincaré group in Fock space. The generators of this group determine physical quantities like energy, momentum, mass, and spin of the photon.

\section{Shifts in spacetime}

A shift with vector $x$ in spacetime maps the vector potentials $A_{\mu}(q)$ onto vector potentials $A_{\mu}^{x}(q)$ given by

$$
A_{\mu}^{x}(q)=A_{\mu}(q-x) .
$$

Using (16), one finds that the Fourier coefficients $a_{\mu}$ transform into $a_{\mu}^{x}$ given by

$$
a_{\mu}^{x}(\mathbf{k})=\exp \left(i|\mathbf{k}| x_{0}-i \mathbf{k} \cdot \mathbf{x}\right) a_{\mu}(\mathbf{k}) .
$$


The corresponding transformation of the classical wave functions $\psi_{\mu}(\mathbf{k})$ is

$$
\psi_{\mu}^{x}(\mathbf{k})=\exp \left(i|\mathbf{k}| x_{0}-i \mathbf{k} \cdot \mathbf{x}\right) \psi_{\mu}(\mathbf{k}) .
$$

With this choice of action the correlation functions $\mathcal{F}(a, \psi ; b, \phi)$ are invariant under shifts. This is what we want because the corresponding state of the system is the vacuum state. Note that the smeared-out fields $f(A)$ are invariant under shifts.

A unitary representation of the group of shifts $\mathbf{R}^{4},+$ is defined by

$$
\hat{U}(x) \hat{W}(a, \psi) \Omega=\hat{W}\left(a^{x}, \psi^{x}\right) \Omega
$$

(see Appendix E). The generators of this representation are denoted $\hat{K}_{\mu}$ and are defined by

$$
\hat{U}(x)=\exp \left(-i x^{\mu} \hat{K}_{\mu}\right) .
$$

By convention, the momentum operators $P_{\mu}$ equal $\hbar \hat{K}_{\mu}$. The energy operator is $c P_{0}=c \hbar \hat{K}_{0}$. The vector $\Omega$ corresponds with the vacuum state and is invariant under shifts. In particular, $\hat{K}_{\mu} \Omega=0$ holds. Hence the energy and momentum of the vacuum are zero, contrary to what is claimed in textbooks, based on the harmonic oscillator picture of the photon.

\section{Energy and momentum of a one-photon state}

Let us calculate

$$
\begin{aligned}
& \left\langle\hat{W}(b, \phi)^{*} \Omega \mid \hat{K}_{0} \hat{W}(a, \psi)^{*} \Omega\right\rangle \\
= & \left.i \frac{\partial}{\partial x_{0}}\right|_{x=0}\left\langle\hat{W}(b, \phi)^{*} \Omega \mid \hat{U}(x) \hat{W}(a, \psi)^{*} \Omega\right\rangle \\
= & \left.i \frac{\partial}{\partial x_{0}}\right|_{x=0} \mathcal{F}\left(a^{x}, \psi^{x} ; b, \phi\right) \\
= & -\left.\mathcal{F}(a, \psi ; b, \phi) i \frac{\partial}{\partial x_{0}}\right|_{x=0}\left(\frac{i}{2 \eta} \operatorname{Im}\left\langle b+i \phi \mid a^{x}+i \psi^{x}\right\rangle\right. \\
& \left.+\frac{1}{4 \eta}\left\langle b-a^{x}+i \eta\left(\phi-\psi^{x}\right) \mid b-a^{x}+i \eta\left(\phi-\psi^{x}\right)\right\rangle\right) \\
= & \frac{1}{2 \eta} \mathcal{F}(a, \psi ; b, \phi)\langle a+i \eta \psi|| \mathbf{k} \mid(b+i \eta \phi)\rangle .
\end{aligned}
$$

Similarly, one shows that, with $\alpha=1,2,3$,

$$
\left\langle\hat{W}(b, \phi)^{*} \Omega \mid \hat{K}_{\alpha} \hat{W}(a, \psi)^{*} \Omega\right\rangle=\frac{1}{2 \eta} \mathcal{F}(a, \psi ; b, \phi)\left\langle a+i \eta \psi \mid \mathbf{k}_{\alpha}(b+i \eta \phi)\right\rangle .
$$

Consider now a one-photon state. From (59) and (60) it follows that

$$
K_{0} \hat{A}_{+}(\psi) \Omega=\hat{A}_{+}(|\mathbf{k}| \psi) \Omega \quad \text { and } \quad K_{\alpha} \hat{A}_{+}(\psi) \Omega=\hat{A}_{+}\left(\mathbf{k}_{\alpha} \psi\right) \Omega
$$


To see this, use that

$$
\left\langle W(b, \phi)^{*} \mid A_{+}(\psi) \Omega\right\rangle=\frac{1}{2}\langle\psi \mid b+i \eta \phi\rangle \mathcal{F}(0,0 ; b, \phi) .
$$

Here we work with photon states smeared out with classical wave functions. It is tradition to associate the notion of photon with states that are not smeared out. These are idealized states which are not represented by wave functions in Hilbert space. In order to approach such a photon state we have to select classical wave functions that converge to a Dirac measure concentrated at a single wave vector $\mathbf{k}$. The energy of such a photon is then equal to $\hbar c|\mathbf{k}|$, the momentum is equal to $\hbar \mathbf{k}$. In particular, this implies that the mass of such an idealized photon is exactly equal to zero.

\section{Lorentz transformations}

The discussion of Lorentz transformations is not very easy because both Fourier coefficients $a_{\mu}(\mathbf{k})$ and classical wave functions $\psi_{\mu}(\mathbf{k})$ depend on a wave vector $\mathbf{k}$ in $\mathbf{R}^{3}$, instead of covariant vectors in $\mathbf{R}^{4}$, and do not obey easy transformation rules. However, both the vector potential $A_{\mu}(q)$ and the test functions $f_{\mu}(q)$ transform as vectors so that the smeared-out vector potential $f(A)$ is invariant under Lorentz transformations. Since $f(A)=-\operatorname{Re}\langle a \mid \psi\rangle$ holds, this shows that the pseudo-scalar product is invariant under Lorentz transformations. Hence the correlation functions (30) are invariant under Lorentz transformations.

Let $\Lambda$ denote a proper Lorentz transformation. Under its action the vector potential $A_{\mu}(q)$ transforms into $A_{\mu}^{\prime}(q)$ given by

$$
A_{\mu}^{\prime}(q)=\Lambda_{\mu}^{\nu} A_{\nu}\left(\Lambda^{-1} q\right) .
$$

Assume first that $\Lambda$ is a spatial rotation described by the 3 -by-3 matrix $R$. Then the Fourier coefficients $a_{\mu}(\mathbf{k})$ transform into $a_{\mu}^{\prime}(\mathbf{k})$ given by

$$
a_{\mu}^{\prime}(\mathbf{k})=\Lambda_{\mu}^{\nu} a_{\nu}(R \mathbf{k}) .
$$

Next consider a boost in direction 3. The non-zero matrix elements are $\Lambda_{00}=$ $\Lambda_{33}=\cosh (\chi), \Lambda_{03}=\Lambda_{30}=\sinh (\chi), \Lambda_{11}=\Lambda_{22}=1$. Then one obtains

$$
\begin{aligned}
& a_{0}^{\prime}(\mathbf{k})=\cosh (\chi) a_{0}\left(\mathbf{k}^{\prime}\right)-\sinh (\chi) a_{3}\left(\mathbf{k}^{\prime}\right) \\
& a_{1}^{\prime}(\mathbf{k})=a_{1}\left(\mathbf{k}^{\prime}\right) \\
& a_{2}^{\prime}(\mathbf{k})=a_{2}\left(\mathbf{k}^{\prime}\right) \\
& a_{3}^{\prime}(\mathbf{k})=-\sinh (\chi) a_{0}\left(\mathbf{k}^{\prime}\right)+\cosh (\chi) a_{3}\left(\mathbf{k}^{\prime}\right)
\end{aligned}
$$

with

$$
\mathbf{k}^{\prime}=\left(\mathbf{k}_{1}, \mathbf{k}_{2}, \cosh (\chi) \mathbf{k}_{3}+\sinh (\chi)|\mathbf{k}|\right) .
$$

Together, the spatial rotations and the boosts in direction 3 generate the proper Lorentz group. Hence, the above formulas represent the action of the proper 
Lorentz group on the Fourier coefficients $a(\mathbf{k})$. The classical wave functions $\psi(\mathbf{k})$ transform in a similar way. A unitary operator $\hat{V}(\Lambda)$ is now defined by

$$
\hat{V}(\Lambda) \hat{W}(a, \psi) \Omega=\hat{W}\left(a^{\prime}, \psi^{\prime}\right) \Omega .
$$

These unitary operators form a representation of the proper Lorentz group. To show this one uses the same arguments as in case of the group of shifts.

\section{The generators of spatial rotations}

The six generators of the proper Lorentz group are denoted $\hat{M}_{\mu \nu}=-\hat{M}_{\nu \mu}$. Three of them correspond with spatial rotations, the other three with boosts.

Consider now a rotation by an angle $\chi$ around the third coordinate axis. The Fourier coefficients transform like

$$
\begin{aligned}
& a_{0}^{\prime}(\mathbf{k})=a_{0}\left(\mathbf{k}^{\prime}\right) \\
& a_{1}^{\prime}(\mathbf{k})=\cos (\chi) a_{1}\left(\mathbf{k}^{\prime}\right)-\sin (\chi) a_{2}\left(\mathbf{k}^{\prime}\right) \\
& a_{2}^{\prime}(\mathbf{k})=\sin (\chi) a_{1}\left(\mathbf{k}^{\prime}\right)+\cos (\chi) a_{2}\left(\mathbf{k}^{\prime}\right) \\
& a_{3}^{\prime}(\mathbf{k})=a_{3}\left(\mathbf{k}^{\prime}\right)
\end{aligned}
$$

with

$$
\mathbf{k}^{\prime}=\left(\cos (\chi) \mathbf{k}_{1}+\sin (\chi) \mathbf{k}_{2},-\sin (\chi) \mathbf{k}_{1}+\cos (\chi) \mathbf{k}_{2}, \mathbf{k}_{3}\right) .
$$

We calculate

$$
\begin{aligned}
& \left\langle\hat{W}(b, \phi)^{*} \Omega \mid \hat{M}_{12} \hat{W}(a, \psi)^{*} \Omega\right\rangle \\
= & \left.i \frac{\partial}{\partial \chi}\right|_{\chi=0}\left\langle\hat{W}(b, \phi)^{*} \Omega \mid V(\Lambda) \hat{W}(a, \psi)^{*} \Omega\right\rangle \\
= & \left.i \frac{\partial}{\partial \chi}\right|_{\chi=0} \mathcal{F}\left(a^{\prime}, \psi^{\prime} ; b, \phi\right) \\
= & -\left.\frac{i}{2 \eta} \mathcal{F}(a, \psi ; b, \phi) \frac{\partial}{\partial \chi}\right|_{\chi=0} \\
& \left(i \operatorname{Im}\left\langle b+i \eta \phi \mid a^{\prime}+i \eta \psi^{\prime}\right\rangle+\frac{1}{2}\left\langle b-a^{\prime}+i \eta\left(\phi-\psi^{\prime}\right) \mid b-a^{\prime}+i \eta\left(\phi-\psi^{\prime}\right)\right\rangle\right) \\
= & \frac{1}{2 \eta} \mathcal{F}(a, \psi ; b, \phi)\left\langle a+i \eta \psi \mid\left(S_{12}+L_{12}\right)(b+i \eta \phi)\right\rangle
\end{aligned}
$$

with $S_{\mu \nu}$ the 4-by-4-matrix with $i$ at position $\mu, \nu,-i$ at position $\nu, \mu$, and zeroes everywhere else, and with

$$
L_{12}=i\left(\mathbf{k}_{1} \frac{\partial}{\partial \mathbf{k}_{2}}-\mathbf{k}_{2} \frac{\partial}{\partial \mathbf{k}_{1}}\right) .
$$




\section{The generators of boosts}

Now let $\Lambda$ be a boost in direction 3 , as given by (65). The corresponding generator $M_{03}$ is calculated as follows.

$$
\begin{aligned}
& \left\langle\hat{W}(b, \phi)^{*} \Omega \mid \hat{M}_{03} \hat{W}(a, \psi)^{*} \Omega\right\rangle \\
= & -\left.i \frac{\partial}{\partial \chi}\right|_{\chi=0}\left\langle\hat{W}(b, \phi)^{*} \Omega \mid V(\Lambda) \hat{W}(a, \psi)^{*} \Omega\right\rangle \\
= & -\left.i \frac{\partial}{\partial \chi}\right|_{\chi=0} \mathcal{F}\left(a^{\prime}, \psi^{\prime} ; b, \phi\right) \\
= & -\left.\frac{i}{2 \eta} \mathcal{F}(a, \psi ; b, \phi) \frac{\partial}{\partial \chi}\right|_{\chi=0} \\
& \left(-i \operatorname{Im}\left\langle b+i \eta \phi \mid a^{\prime}+i \eta \psi^{\prime}\right\rangle-\frac{1}{2}\left\langle b-a^{\prime}+i \eta\left(\phi-\psi^{\prime}\right) \mid b-a^{\prime}+i \eta\left(\phi-\psi^{\prime}\right)\right\rangle\right) \\
= & -\frac{1}{2 \eta} \mathcal{F}(a, \psi ; b, \phi)\left\langle a+i \eta \psi \mid\left(S_{03}-L_{03}\right)(b+i \eta \phi)\right\rangle .
\end{aligned}
$$

with

$$
L_{0 \alpha}=i|\mathbf{k}| \frac{\partial}{\partial \mathbf{k}_{\alpha}}
$$

\section{Spin of the photon}

From expressions $(70,72)$ it is clear that there are two different types of contributions to the generators $M_{\mu \nu}$ of the Lorentz group. These are called the spin part $\hat{S}$, respectively the orbital part $\hat{L}$. The spin contribution originates from the vector character of the electromagnetic vector potential $A_{\mu}(q)$, the orbital part follows from the transformation of Minkowski space. The operators $\hat{L}_{23}$, $\hat{L}_{31}$ and $\hat{L}_{12}$ are the components of angular momentum, the operators $\hat{S}_{23}, \hat{S}_{31}$ and $\hat{S}_{12}$ are the components of the spin of the photon. Note that these notions are not covariant. Worse is that the splitting of $\hat{M}$ into $\hat{S}$ and $\hat{L}$ is not gauge invariant. This implies that the components of $\hat{S}$ and $\hat{L}$ are not physically observable. Hence one could say that the mechanical spin of the photon is not observable. See Jauch and Rohrlich[2] for a discussion of these points.

However, it is common to say that the photon is a spin-1 particle. In order to understand this statement let us assume that a one-photon state $\hat{A}_{+}(\psi) \Omega$ is an eigenstate of the operator $\hat{S}_{12}$

$$
\hat{S}_{12} \hat{A}_{+}(\psi) \Omega=\lambda \hat{A}_{+}(\psi) \Omega .
$$

From

$$
\left\langle\hat{W}(b, \phi)^{*} \Omega \mid \hat{S}_{12} \hat{W}(a, \psi)^{*} \Omega\right\rangle=\frac{1}{2 \eta} \mathcal{F}(a, \psi ; b, \phi)\left\langle a+i \eta \psi \mid S_{12}(b+i \eta \phi)\right\rangle
$$

follows

$$
\left\langle\hat{W}(b, \phi)^{*} \Omega \mid \hat{S}_{12} A_{+}(\psi) \Omega\right\rangle=\frac{1}{2}\left\langle\hat{W}(b, \phi)^{*} \Omega \mid \Omega\right\rangle\left\langle\psi \mid S_{12}(b+i \eta \phi)\right\rangle .
$$


In combination with the assumption that the one-photon state is an eigenstate of $\hat{S}_{12}$ with eigenvalue $\lambda$ there follows

$$
\lambda\left\langle\hat{W}(b, \phi)^{*} \Omega \mid A_{+}(\psi) \Omega\right\rangle=\frac{1}{2}\left\langle\hat{W}(b, \phi)^{*} \Omega \mid \Omega\right\rangle\left\langle\psi \mid S_{12}(b+i \eta \phi)\right\rangle .
$$

On the other hand is

$$
\left\langle\hat{W}(b, \phi)^{*} \Omega \mid A_{+}(\psi) \Omega\right\rangle=\frac{1}{2}\left\langle\hat{W}(b, \phi)^{*} \Omega \mid \Omega\right\rangle\langle\psi \mid b+i \eta \phi\rangle .
$$

Comparison of both expressions gives the condition

$$
S_{12} \psi(\mathbf{k})=\lambda \psi(\mathbf{k})
$$

Now, the eigenvalues of the matrix $S_{12}$ are $+1,-1$, and 0 (two-fold degenerated). Hence, the space of classical wave functions $H$ can be split into three real-linear subspaces $H_{+}, H_{0}$, and $H_{-}$with the properties that $S_{12} \psi= \pm \psi$ if $\psi$ is in $H_{+}$, respectively in $H_{-}$, and $S_{12} \psi=0$ if $\psi$ is in $H_{0}$. As a consequence, also the onephoton subspace of Fock space can be written as a direct sum of three subspaces which consist of eigenvectors of $\hat{S}_{12}$ corresponding to the eigenvalues $+1,0,-1$. Spin-operators with a spectrum $+1,0,-1$ are associated with spin-1 particles.

\section{Polarization of the photon}

As stated earlier in section 2, each class of equivalent classical wave functions contains a representative $\psi$ satisfying $\psi_{0}=0$. None of these representatives belongs to $H_{0}$. Indeed, if $S_{12} \psi(\mathbf{k})=0$ holds for all $\mathbf{k}$ then $\psi_{1}=\psi_{2}=0$ and $|\mathbf{k}| \psi_{0}(\mathbf{k})=\mathbf{k}_{3} \psi_{3}(\mathbf{k})$. But because of $\psi_{0}=0$ also $\psi_{3}=0$ follows. Hence, if a representative $\psi$ belongs to $H_{0}$ then all of its components are zero. Classical wavefunctions in $H_{ \pm}$satisfy

$$
0=\left(\mathbf{k}_{1} \mp i \mathbf{k}_{2}\right) \psi_{1}(\mathbf{k})=\mathbf{k}_{3} \psi_{3}(\mathbf{k}) \quad \text { and } \psi_{2}(\mathbf{k})=\mp i \psi_{1}(\mathbf{k})
$$

with $\psi_{1}(\mathbf{k})$ and $\psi_{2}(\mathbf{k})$ not identically zero. The only solutions of these conditions are idealized photons with wave vector parallel to the third direction and with $\psi_{3}(\mathbf{k})=0$. This implies that the electric and magnetic fields lie in the plane orthogonal to the wave vector $\mathbf{k}$. Two independent solutions are allowed. They correspond with the two independent polarizations of the electromagnetic field. A more detailed analysis can be found in Jauch and Rohrlich[2].

\section{Conclusions}

We have shown in this paper that the standard theory of the free photon field can be derived within the covariance approach to quantum mechanics. Typical for this approach is that it starts from the action of a group in a $C^{*}$-algebra and from correlation functions describing a state of the covariance system. In 
the case of the free radiation field the $C^{*}$ - algebra is the algebra of complex numbers, the group is the group of adding fields times the group of adding test functions. The Lorenz gauge is used to eliminate part of the redundancy. The action is trivial. The correlation functions describe a Lorentz invariant vacuum state. The state vectors of the induced Fock representation are invariant under the remaining gauge freedoms.

The present approach has several advantages. In the first place the formalism is mathematically rigorous. The development of photon theory is crystal clear and there is no need for hand waving arguments. Both the gauge problem and the problem of positivity of the scalar product are solved in a satisfactory manner. The approach is generic. It is obvious how to apply it to other fields than the electromagnetic one.

We have stressed that there exists a duality between classical wave functions $\psi$ and Fourier coefficients $a$. As a consequence of this duality there exist, besides the usual field operators $\hat{A}(\psi)$, also operators $\hat{F}(a)$, labeled with Fourier transformed vector potentials. However, in the representation of the electromagnetic vacuum state the field operators $\hat{F}(a)$ and $\hat{A}(\psi)$ coincide. Hence, this duality has no practical consequences for the description of the vacuum. We do not know if this degeneracy continues to exist in other representations of the electromagnetic radiation field.

The formalism considers photons smeared out with classical wave functions. These differ from the idealized photons discussed in most text books. The reason for smearing-out is of course that the strictly localized objects $\hat{A}(q)$ cannot be defined as operators in Fock space, while the smeared-out equivalents $\hat{A}(\psi)$ are nicely defined self-adjoint operators. Finally, the generators of the Lorentz group can be decomposed into a sum of an orbital part and a spin part. The space of classical wave functions can be split into three parts corresponding with spin 1, 0, and -1 respectively. Because of gauge freedom only two independent polarizations of the idealized photons occur.

Up to now, we did not consider electromagnetic fields in presence of external charges and currents. The first question that arises in this context is whether all states of the covariance system of the free radiation field (i.e. the one used in the present paper) describe radiation fields, or whether states can be found which describe fields produced by charges and currents. If the latter is not the case, then the covariance system has to be modified. Another topic for further investigation is the description of massive photons in terms of the present formalism (see e.g. section 6-5 of Jauch and Rohrlich [2]). Our ultimate goal is of course a combination of electron and photon fields within the same covariance approach.

\section{Acknowledgement}

We thank Marek Czachor for his interest in the present work. 


\section{Appendix A: Covariance systems}

A covariance system $(\mathcal{A}, X, \sigma)$ consists of a $C^{*}$-algebra $\mathcal{A}$, a locally compact group $X$, and an action $\sigma$ of this group as automorphisms of $\mathcal{A}$. For each $a \in \mathcal{A}$ the map $x \in X \rightarrow \sigma_{x} a$ should be continuous. In the present paper, the $C^{*}$-algebra $\mathcal{A}$ is the algebra $\mathbf{C}$ of complex numbers. In this case the only possible action of $X$ is the trivial one, leaving the complex numbers invariant. The resulting covariance system is denoted $(\mathbf{C}, X, \mathbf{I})$ and is rather trivial. Still, the notions of state and of representation of a covariance system apply, and are nontrivial.

A state[7] of a covariance system $(\mathcal{A}, X, \sigma)$ is determined by correlation functions $\mathcal{F}(a, x, y)$ depending on $a \in \mathcal{A}$ and $x, y \in X$. They satisfy conditions of positivity, normalization, covariance, and continuity. In the present context, where the $C^{*}$-algebra is the algebra of complex numbers, the dependence on elements of $\mathcal{A}$ can be omitted, and the conditions reduce to

- (positivity) For all $n>0$ and for all possible choices of $\lambda_{1}, \ldots, \lambda_{n}$ in $\mathbf{C}$, of $x_{1}, \ldots, x_{n}$ in $X$, is

$$
\sum_{j, k=1}^{n} \lambda_{j} \overline{\lambda_{k}} \mathcal{F}\left(x_{j}, x_{k}\right) \geq 0
$$

- (normalization) $\mathcal{F}(e, e)=1$ ( $e$ is the neutral element of $X)$.

- $($ covariance) $\mathcal{F}(x z, y z)=\mathcal{F}(x, y)$ for all $x, y, z$ in $X$.

- (continuity) the map $x, y \rightarrow \mathcal{F}(x, y)$ is continuous in a neighborhood of the neutral element of $X$.

A representation of the covariance system $(\mathbf{C}, X, \mathbf{I})$ is nothing but a projective representation $U$ of the group $X$ as unitary operators of a Hilbert space $\mathcal{H}$, with the property that the map $x \rightarrow U(x)$ is strongly continuous for $x$ in a neighborhood of the neutral element of $X$. In this context the generalized G.N.S.-theorem states that for each state of $(\mathbf{C}, X, \mathbf{I})$, described by the correlation functions $\mathcal{F}(x, y)$, there exists a representation $U$ of $(\mathbf{C}, X, \mathbf{I})$ in a Hilbert space $\mathcal{H}$ and an element $\Omega$ of $\mathcal{H}$ with the property that

$$
\mathcal{F}(x, y)=\left\langle U(y)^{*} \Omega \mid U(x)^{*} \Omega\right\rangle
$$

holds for all $x$ and $y$ in $X$.

\section{Appendix B: Smeared-out field operators}

Here we discuss the relation between expressions (11) and (3). The obvious relation between $\hat{A}(\psi)$ and $\hat{A}_{\mu}(q)$ is

$$
\hat{A}(\psi)=\int_{\mathbf{R}^{4}} \mathrm{~d} q f^{\mu}(q) \hat{A}_{\mu}(q) .
$$


where the test functions $f_{\mu}$ correspond with classical wavefunctions $\psi_{\mu}$ by $(20)$. Similarly, let $\phi_{\mu}$ correspond with test functions $g_{\mu}$. Then one obtains, using the commutation relations (11) and definition (2) of $D_{0}(q)$,

$$
\begin{aligned}
{[\hat{A}(\psi), \hat{A}(\phi)]=} & \int_{\mathbf{R}^{4}} \mathrm{~d} q \int_{\mathbf{R}^{4}} \mathrm{~d} q^{\prime} f^{\mu}(q) g^{\nu}\left(q^{\prime}\right)\left[\hat{A}_{\mu}(q), \hat{A}_{\nu}\left(q^{\prime}\right)\right] \\
= & -i \int_{\mathbf{R}^{4}} \mathrm{~d} q \int_{\mathbf{R}^{4}} \mathrm{~d} q^{\prime} f^{\mu}(q) g_{\mu}\left(q^{\prime}\right) D_{0}\left(q-q^{\prime}\right) \\
= & -i \frac{1}{(2 \pi)^{3}} \int_{\mathbf{R}^{4}} \mathrm{~d} q \int_{\mathbf{R}^{4}} \mathrm{~d} q^{\prime} f^{\mu}(q) g_{\mu}\left(q^{\prime}\right) \\
& \times \int_{\mathbf{R}^{3}} \mathrm{~d} \mathbf{k} \exp \left(i \mathbf{k} \cdot\left(\mathbf{q}-\mathbf{q}^{\prime}\right)\right) \frac{1}{|\mathbf{k}|} \sin \left(\left(q_{0}-q_{0}^{\prime}\right)|\mathbf{k}|\right) \\
= & -\frac{1}{(2 \pi)^{3}} \int_{\mathbf{R}^{3}} \mathrm{~d} \mathbf{k} \frac{1}{2|\mathbf{k}|} \int_{\mathbf{R}^{4}} \mathrm{~d} q f^{\mu}(q) \exp (i \mathbf{k} \cdot \mathbf{q}) \\
& \times \int_{\mathbf{R}^{4}} \mathrm{~d} q^{\prime} g_{\mu}\left(q^{\prime}\right) \exp \left(-i \mathbf{k} \cdot \mathbf{q}^{\prime}\right) \\
& \times\left(\exp \left(i\left(q_{0}-q_{0}^{\prime}\right)|\mathbf{k}|\right)-\exp \left(-i\left(q_{0}-q_{0}^{\prime}\right)|\mathbf{k}|\right)\right) .
\end{aligned}
$$

Using the definition (20) of classical wave functions one obtains

$$
[\hat{A}(\psi), \hat{A}(\phi)]=-\int_{\mathbf{R}^{3}} \mathrm{~d} \mathbf{k} \frac{1}{2|\mathbf{k}|}\left(\psi^{\mu}(\mathbf{k}) \overline{\phi_{\mu}(\mathbf{k})}-\overline{\psi^{\mu}(\mathbf{k})} \phi_{\mu}(\mathbf{k})\right) .
$$

The latter implies (3).

\section{Appendix C: Representative classical wave func- tions}

Here we show that each class of equivalent classical wave functions contains a representative satisfying the radiation gauge. Given the classical wave functions $\psi_{\mu}(\mathbf{k})$, let

$$
\lambda(\mathbf{k})=\frac{1}{|\mathbf{k}|^{2}} \sum_{\alpha=1}^{3} \mathbf{k}_{\alpha} \psi_{\alpha}(\mathbf{k})
$$

and

$$
\phi_{0}(\mathbf{k})=0, \quad \phi_{\alpha}(\mathbf{k})=\psi_{\alpha}(\mathbf{k})-\lambda(\mathbf{k}) \mathbf{k}_{\alpha}, \quad \alpha=1,2,3 .
$$

Then one calculates

$$
\sum_{\alpha=1}^{3} \mathbf{k}_{\alpha} \phi_{\alpha}(\mathbf{k})=\sum_{\alpha=1}^{3} \mathbf{k}_{\alpha} \psi_{\alpha}(\mathbf{k})-\lambda(\mathbf{k})|\mathbf{k}|^{2}=0 .
$$

Hence $\phi_{\mu}(\mathbf{k})$ are classical wave functions satisfying the radiation gauge.

Consider now an arbitrary set of Fourier coefficients $a_{\mu}(\mathbf{k})$ satisfying (19). Then one finds

$$
\langle a \mid \phi\rangle=\int_{\mathbf{R}^{3}} \mathrm{~d} \mathbf{k} \frac{1}{2|\mathbf{k}|} \sum_{\alpha=1}^{3} \overline{a_{\alpha}(\mathbf{k})} \phi_{\alpha}(\mathbf{k})
$$




$$
\begin{aligned}
= & \int_{\mathbf{R}^{3}} \mathrm{~d} \mathbf{k} \frac{1}{2|\mathbf{k}|} \sum_{\alpha=1}^{3} \overline{a_{\alpha}(\mathbf{k})} \psi_{\alpha}(\mathbf{k}) \\
& -\int_{\mathbf{R}^{3}} \mathrm{~d} \mathbf{k} \frac{1}{2|\mathbf{k}|} \lambda(\mathbf{k}) \sum_{\alpha=1}^{3} \overline{a_{\alpha}(\mathbf{k})} \mathbf{k}_{\alpha} .
\end{aligned}
$$

Using (19) this becomes

$$
\begin{aligned}
\langle a \mid \phi\rangle= & \int_{\mathbf{R}^{3}} \mathrm{~d} \mathbf{k} \frac{1}{2|\mathbf{k}|} \sum_{\alpha=1}^{3} \overline{a_{\alpha}(\mathbf{k})} \psi_{\alpha}(\mathbf{k}) \\
& -\frac{1}{2} \int_{\mathbf{R}^{3}} \mathrm{~d} \mathbf{k} \lambda(\mathbf{k}) \overline{a_{0}(\mathbf{k})} \\
= & \int_{\mathbf{R}^{3}} \mathrm{~d} \mathbf{k} \frac{1}{2|\mathbf{k}|} \sum_{\alpha=1}^{3} \overline{a_{\alpha}(\mathbf{k})} \psi_{\alpha}(\mathbf{k}) \\
& -\int_{\mathbf{R}^{3}} \mathrm{~d} \mathbf{k} \frac{1}{2|\mathbf{k}|^{2}} \overline{a_{0}(\mathbf{k})} \sum_{\alpha=1}^{3} \mathbf{k}_{\alpha} \psi_{\alpha}(\mathbf{k}) \\
= & \langle a \mid \psi\rangle .
\end{aligned}
$$

To obtain the latter, (24) has been used. This shows that $\phi$ and $\psi$ are equivalent.

\section{Appendix D: Existence of the vacuum state}

The difficult point to verify is positivity of the state. Note that the symbols $\psi_{j}$ and $a_{j}$ below have each 4 components $\left(\psi_{j}\right)_{\mu}$ respectively $\left(a_{j}\right)_{\mu}$. One verifies, using the notation $b_{j}=a_{j}+i \psi_{j}$,

$$
\begin{aligned}
\sum_{j j^{\prime}} \lambda_{j} \overline{\lambda_{j^{\prime}}} \mathcal{F}\left(a_{j}, \psi_{j} ; a_{j^{\prime}} \psi_{j^{\prime}}\right)= & \sum_{j j^{\prime}} \lambda_{j} \overline{\lambda_{j^{\prime}}} \exp \left(-\frac{i}{2 \eta} \operatorname{Im}\left\langle b_{j^{\prime}} \mid b_{j}\right\rangle\right) \\
& \times \exp \left(-\frac{1}{4 \eta}\left\langle\left(b_{j^{\prime}}-b_{j}\left|b_{j^{\prime}}-b_{j}\right\rangle\right)\right.\right. \\
= & \sum_{j j^{\prime}} \mu_{j} \overline{\mu_{j^{\prime}}} \exp \left(\frac{1}{2 \eta}\left\langle b_{j} \mid b_{j^{\prime}}\right\rangle\right)
\end{aligned}
$$

with

$$
\mu_{j}=\lambda_{j} \exp \left(-(1 / 4 \eta)\left\langle b_{j} \mid b_{j}\right\rangle\right) .
$$

Positivity of (91) follows by means of Schur's lemma, provided we can show that the matrix with elements $\left\langle b_{j} \mid b_{j^{\prime}}\right\rangle$ is positive-definite. But the latter is clear because of the positivity of the scalar product, for which we now give a proof. Using the Lorenz condition (19, 24), one writes

$$
\langle\phi \mid \phi\rangle=\int_{\mathbf{R}^{3}} \mathrm{~d} \mathbf{k} \frac{1}{2|\mathbf{k}|}\left(-\overline{\phi_{0}(\mathbf{k})} \phi_{0}(\mathbf{k})+\sum_{\alpha=1}^{3} \overline{\phi_{\alpha}(\mathbf{k})} \phi_{\alpha}(\mathbf{k})\right)
$$




$$
\begin{aligned}
& =\int_{\mathbf{R}^{3}} \mathrm{~d} \mathbf{k} \frac{1}{2|\mathbf{k}|^{3}} \sum_{\alpha, \beta=1}^{3} \overline{\phi_{\alpha}(\mathbf{k})}\left[|\mathbf{k}|^{2} \delta_{\alpha, \beta}-\mathbf{k}_{\alpha} \mathbf{k}_{\beta}\right] \phi_{\beta}(\mathbf{k}) \\
& \geq 0 .
\end{aligned}
$$

The latter follows because the matrix $|\mathbf{k}|^{2} \delta_{\alpha, \beta}-\mathbf{k}_{\alpha} \mathbf{k}_{\beta}$ is positive-definite.

\section{Appendix E: Unitary representation of the group of shifts}

We show here that a unitary representation of the group of shifts $\mathbf{R}^{4},+$ is determined by (57).

Let us show that $U(x)$ is well-defined. Assume that

$$
\hat{W}\left(a^{x}, \psi^{x}\right) \Omega=\hat{W}\left(b^{x}, \phi^{x}\right) \Omega .
$$

By taking the inner product with $\hat{W}(c, \chi)^{*} \Omega$ one obtains

$$
\mathcal{F}\left(a^{x}, \psi^{x} ; c, \chi\right)=\mathcal{F}\left(b^{x}, \phi^{x} ; c, \chi\right) .
$$

Note now that $\left\langle a^{x} \mid \psi^{x}\right\rangle=\langle a \mid \psi\rangle$ so that

$$
\mathcal{F}\left(a^{x}, \psi^{x} ; c, \chi\right)=\mathcal{F}\left(a, \psi ; c^{-x}, \chi^{-x}\right) .
$$

Hence (95) becomes

$$
\mathcal{F}\left(a, \psi ; c^{-x}, \chi^{-x}\right)=\mathcal{F}\left(b, \phi ; c^{-x}, \chi^{-x}\right) .
$$

This implies

$$
\left\langle\hat{W}\left(c^{-x}, \chi^{-x}\right)^{*} \Omega \mid\left(\hat{W}(a, \psi)^{*}-\hat{W}(b, \phi)^{*}\right) \Omega\right\rangle=0 .
$$

Since $c$ and $\chi$ are arbitrary, it follows that $\hat{W}(a, \psi)^{*} \Omega=\hat{W}(b, \phi)^{*} \Omega$. This shows that $U(x)$ is well-defined.

It is now straightforward to show that $U(x) U(y)=U(x+y)$ and that $U(x)$ is isometric. Therefore, $U(x)$ is a unitary representation of $\mathbf{R}^{4},+$. 


\section{References}

[1] A.L. Carey, J.M. Gaffney, and C.A.Hurst, A $C^{*}$-algebra formulation of the quantization of the electromagnetic field, J. Math. Phys. 18, 629-640 (1977).

[2] J.M. Jauch and F. Rohrlich, The theory of photons and electrons, 2nd ed. (Springer-Verlag, 1980).

[3] G. Scharf, Finite quantum electrodynamics (Springer-Verlag, 1989).

[4] D. Petz, An Invitation to the Algebra of Canonical Commutation Relations (Leuven University Press, Leuven, 1990)

[5] S. Doplicher, K. Fredenhagen, J.E. Roberts, Spacetime quantization induced by classical gravity, Phys. Lett. B331, 39-44 (1994).

[6] S. Doplicher, K. Fredenhagen, J.E. Roberts, The Quantum Structure of Spacetime at the Planck Scale and Quantum Fields, Commun. Math. Phys. 172, 187-220 (1995)

[7] J. Naudts, M. Kuna, Covariance systems, math-ph/0009031, J. Phys. A: Math. Gen. 34, 9265-9280 (2001).

[8] J. Naudts, M. Kuna, Model of a particle in spacetime, J. Phys. A: Math. Gen. 34, 4227-4239 (2001).

[9] J. Naudts, Covariance approach to quantum theory, proceedings of the conference "Quantum Theory and Symmetries", Krakow, July 2001. 